\title{
Development of radioimmunoconjugate for diagnosis and management of head-and-neck subclinical cancer and colorectal carcinoma
}

\author{
Raquel Benedetto ${ }^{1 *}$, Adriana Vidal Fernandes Massicano ${ }^{1}$, Jefferson Jesus Silva1, \\ Cristian Antonio Wieczorek Villas Boas ${ }^{1}$, Jair Mengatti ${ }^{1}$, Elaine Bortoleti de Araújo ${ }^{1}$ \\ ${ }^{1}$ Radiopharmacy Center, IPEN - Institute of Energy and Nuclear Research, São Paulo, Brazil
}

\begin{abstract}
Scientific innovations in diagnostic methods are important drivers of cancer control and prevention. Noninvasive imaging of the epidermal growth factor receptor (EGFR) in head-and-neck squamous, cell carcinoma and colorectal cancer could be valuable to select patients for EGFR-targeted therapy, as well as to monitor the efficacy and occurrence of resistance to immunotherapy. In order to develop the first Brazilian radioimmunoconjugate for diagnosis, Cetuximab has been conjugated to p-SCN-Bn-DTPA chelator and radiolabeled with Indium-111. The conjugation methodology was optimized using different $\mathrm{mAb}$ :DTPA molar ratios, time was then reduced for immunoconjugate preparation, besides the protein recovery' percentage increased after purification $(\mathrm{m}=83.8 \pm 0.91 \%)$. The stability of Cetuximab-DTPA at $-20{ }^{\circ} \mathrm{C}$ was evaluated for six months, and its integrity was greater than $90 \%(\mathrm{~m}=93.9 \pm 1.5 \%, \mathrm{~N}=24)$. The radioimmunoconjugate with specific activity of $185 \mathrm{MBq} / \mathrm{mg}$ showed radiochemical purity above $95 \%(\mathrm{~m}=96.8 \pm 1.31 \%, \mathrm{~N}=15)$. We conclude that the radioimmunoconjugate ${ }^{111}$ In-DTPA-cetuximab is stable and may be applied to the diagnosis of EGFR-positive tumors.
\end{abstract}

Keywords: Radioimmunoconjugate/development. Head and neck cancer/diagnosis. Tumors diagnosis. Cetuximab. SPECT. ${ }^{111}$ In.

\section{INTRODUCTION}

The longevity of the population has contributed to the development of chronic-degenerative diseases, including malignant neoplasm. Colorectal cancer (CRC) is one of the most common malignant tumors in the world. According to the latest global estimate/study, CRC is configured as the third most common type of cancer among men and the second in women (INCA, 2014). In accordance with the World Health Organization estimate/ study, head-and-neck squamous cell carcinoma (HNSCC) is the fifth most common cancer worldwide, with an overall annual rate/occurrence of 980,000 new cases.

Site-specific delivery of therapeutic agents has been an ultimate goal of the pharmaceutical industry in order to maximize drug action and minimize side effects (Mccarron

\footnotetext{
*Correspondence: R. Benedetto. Centro de Radiofarmácia, Instituto de Pesquisas Energéticas e Nucleares - IPEN. Av. Prof. Lineu Prestes, 2242, Cidade Universitária, CEP: 05508-000 - São Paulo - SP - Brasil. E-mail: benedettoraquel@yahoo.com.br
}

et al., 2005). Targeted therapy is becoming an increasingly important component in the treatment of cancer (Hoeben et al., 2011). In this scenario, monoclonal antibodies (mAbs) have been highlighted due to their success in therapeutic application.

The epidermal growth factor receptor (EGFR) is a tyrosine kinase receptor and it is overexpressed in the majority of epithelial malignancies, such as non-small cell lung cancer (NSCLC), epithelial ovarian cancer, including head-and-neck cancer (Hoeben et al., 2011). Furthermore, EGFR overexpression is associated with advanced tumor stage and poor prognosis in several epithelial cancer types. EGFR plays an important role in cellular proliferation, DNA repair and regulation responses to hypoxia - through hypoxia-inducible factor 1 (HIF-1), hypoxia signaling promotes angiogenesis and metastasis by inducing epithelial-mesenchymal-transition (EMT) (Wiechec et al., 2017).

Cetuximab, a chimeric monoclonal antibody (IgG1) against EGFR has been approved by the Food and Drug Administration (FDA) in 2004, as a single drug or in 
combination with: (a) chemotherapy for treatment of colorectal and head-and-neck squamous cell carcinoma or (b) external radiotherapy for treatment of head-andneck squamous cell carcinoma (Sihver et al., 2014). The conjugation of radionuclides to cetuximab, in combination with the specific targeting properties of this antibody, has been performed for imaging. Noninvasive imaging of EGFR in head-and-neck squamous cell carcinoma could be of value to select patients for EGFR-targeted therapy (Hoeben et al., 2011). Molecular imaging (MI), especially single photon emission computed tomography (SPECT) and positron emission tomography (PET), is a powerful tool for detecting cancer and for characterizing the biological properties of tumors throughout the body (Reilly et al., 2015).

Considering the importance of Cetuximab in the immunotherapy of colon cancer and head-and-neck carcinoma, several $\mathrm{mAb}$ labeling studies with different radionuclides have been developed to (1) produce a specific radiopharmaceutical for selecting appropriate candidates for therapy, (2) monitor the effectiveness of immunotherapy, and (3) evaluate drug resistance (Sihver et al., 2014). Cetuximab has been labeled with different radionuclides for diagnosis for instance technetium-99m (Schechter et al., 2004), gallium-68 (Evans et al., 2014) and zirconium-89 (Van de Watering et al., 2014). For therapy, lutetium-177 (Jalilian et al., 2016) and yttrium-90 (Niu et al., 2010) have been used. Ping et al. (2008) reported a preclinical study related to the use of cetuximab as a PET agent, directed to tumors overexpression ' EGFR. Cetuximab was conjugated with DOTA chelator $(1,4,7,10$ - tetraazacyclododecane tetraacetic acid), for radiolabeling with copper-64 ( $\mathrm{T}_{1 / 2}=12.7$ hours $)$.

Indium-111 is primarily a gamma emitter, and a cyclotron-produced radiometal and one of the most commonly used radionuclides for SPECT, especially as a label for mAbs, due to its adequate physical half-life (2.8 d). Thus, pre-clinical and clinical studies have been investigated with ${ }^{111} \mathrm{In}$-labeled $\mathrm{mAb}$ conjugates (Sihver et al., 2014).

Indium-111 radiolabeled Cetuximab was applied in a clinical phase I study in patients with inoperable lung squamous cell carcinoma, which express high levels of EGF receptors. No toxicity was observed. (Divgi et al., 1991).

Others $m A$ bs have been labeled with indium-111 and intended in clinical studies (Doerr et al., 1990, Morris et al., 2005, Perik et al, 2006, Scott et al., 2007). Indium-111 radiolabeled Pertuzumab ( ${ }^{111}$ In-BzDTPA-Pertuzumab) was used to evaluate breast cancer, which responds to the treatment with trastuzumab (Herceptin), in patients with HER2-positive metastatic disease. The clinical images showed good tumor uptake in hepatic metastases 48 hours after injection of $111 \mathrm{MBq}(5 \mathrm{mg})$ of ${ }^{111} \mathrm{In}$-BzDTPAPertuzumab. Furthermore, the study revealed no serious adverse effects (Reilly et al., 2015).

The production of radiolabeled monoclonal antibodies is a process that involves several steps (Cooper, Sabbah, Mather, 2006): (1) pre-purification of $\mathrm{mAb}$ to remove free metals; (2) conjugation of $\mathrm{mAb}$ with chelating groups; (3) immunoconjugate purification and concentration; (4) and radiolabeling.

Considering several steps and many different variables, it was imperative for our laboratory to establish appropriated working conditions to produce ${ }^{111}$ In-DTPACetuximab with high radiochemical yield and stability. In an unprecedented way, this research evaluated the influence of the specific activity $(\mathrm{MBq} / \mathrm{mg})$ on the radiochemical purity and stability of the preparation. The specific activity is an important property for specific targetlabeled radiopharmaceuticals, since the unlabeled antibody will compete with the radiopharmaceutical for the same receptors. On the other hand, high specific activity could increase the effect of radiolysis on antibody structure.

In most countries, the preparation of radioimmunoconjugates is performed "in house", therefore, radiopharmaceuticals for radioimmunodiagnostic (RID) and radioimmunotherapy (RIT) are prepared and applied at the same clinical imaging center. Currently, the production of these radiopharmaceuticals in Brazil is carried out by the Institute of Energy and Nuclear Research (IPEN-CNEN), in São Paulo, which guarantees the radiopharmaceutical's quality and stability during their transport and distribution to the clinical research centers. Therefore, the stability of the radiolabeled $\mathrm{mAb}$ was evaluated, in order to determine the radiopharmaceutical expiration date, and the optimal conditions for storage, that would allow the radioimmunoconjugate to be transported to different sites for future clinical studies.

Considering the first experience in Brazil, this study presents the procedures for ${ }^{111}$ In-DTPA-Cetuximab preparation. We aimed to optimize the conjugation process to obtain a radioimmunoconjugate with high radiochemical yield and stability for future pre-clinical and clinical applications.

\section{MATERIAL AND METHODS}

\section{Analysis of the structural integrity of the antibody}

The Cetuximab (5 mg/mL) (Erbitux, Merck, Darmstadt, Germany) was analyzed by electrophoresis 
(Laemmli, 1970) in order to verify the integrity of the antibody. The electrophoresis was performed in a BioRad system, using a 4-20\% Tris-Glycine gel. Then the antibody was diluted in non-reducing Laemmli buffer and the gel was run at $200 \mathrm{~V}$ for 30 minutes. Afterwards, the gel was analyzed using a Packard instant image electronic autoradiography.

\section{Conjugation of Cetuximab with p-SCN-Bn-DTPA}

The production of radiolabeled monoclonal antibodies involves several steps which have been summarized in Figure 1. Cetuximab was conjugated to p-isothiocyanatobenzyl-diethylenetriaminepentaacetic acid (p-SCN-Bn-DTPA, Macrocyclis, Dallas, TX) in $0.2 \mathrm{M}$ sodium bicarbonate $\mathrm{pH} 8.5$ for 1 hour at room temperature, using 10 and 20 times molar excess of DTPA.

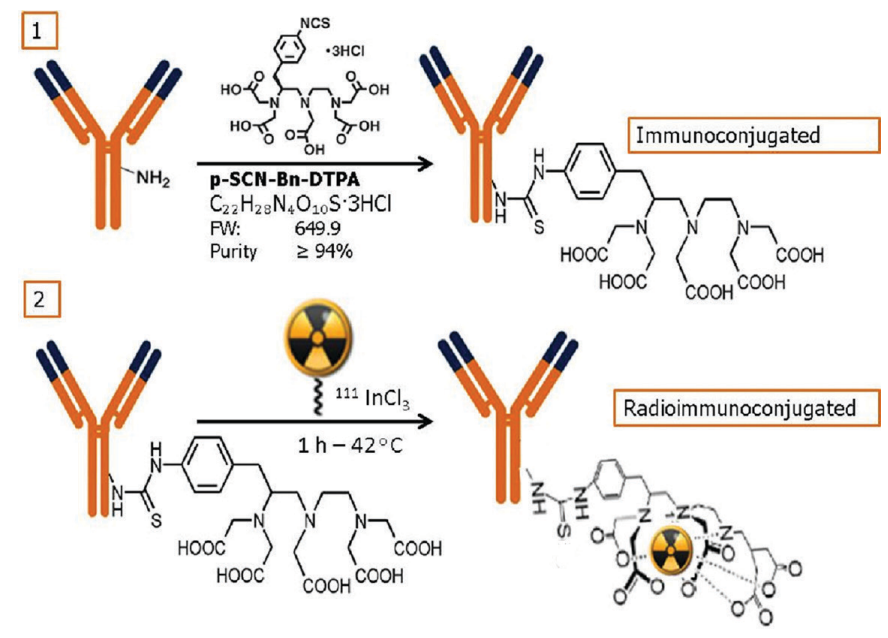

FIGURE 1 - Steps of the radioimmunoconjugate preparation.

\section{Purification of the immunoconjugate}

The conjugation process was carried out with excess chelator which must be removed after conjugation, while avoiding interference with the radiolabeling process. After the conjugation time, ${ }^{111}$ In-DTPA-Cetuximab was purified by molecular exclusion gel column (PD-10 column - GE Healthcare Bio-Sciences, Pittsburgh, USA). The column was equilibrated with $50 \mathrm{~mL}$ of $0.25 \mathrm{M}$ ammonium acetate buffer $\mathrm{pH}$ 6.5. Ten aliquots of $0.5 \mathrm{~mL}$ were collected and the absorbance of each sample was analyzed in a spectrophotometer (160 Evolution - Thermo Scientific, USA, $280 \mathrm{~nm}$ ). All fractions were subjected to analysis by high-performance liquid chromatography (HPLC) (Shimadzu BioSep SEC S-3000 column x $3007.8 \mathrm{~mm} 5$ $\mu \mathrm{m}, 1 \mathrm{~mL} / \mathrm{min}$ flow, mobile phase $0.1 \mathrm{M}$ sodium phosphate buffer $\mathrm{pH} 7.0,15$ minute), with UV detection $(280 \mathrm{~nm})$, to examine the structural integrity of the immunoconjugate. Substances with higher molecular weight are first eluted in a molecular exclusion column, thus, the immunoconjugate has lower retention time (RT) when compared with the unconjugated antibody. The fractions that contained the pure immunoconjugate were selected and subsequently combined in an ultrafiltration concentrator tube (Amicon ${ }^{\circledR}$ Ultra 10,000 MWCO, Millipore, USA) (Mikro 220, Hettich Germany). Statistical analysis was performed using Prism software (GraphPad Software).

\section{Determination of protein concentration of the immunoconjugate}

The protein concentration of the purified immunoconjugate was determined by the bicinchoninic acid method (BCA), using Albumin standard $5 \mathrm{mg} / \mathrm{ml}$ (Thermo Scientific, EUA), as previously described (Brady et al., 2004).

\section{Determination of the number of chelates per antibody by the spectrophotometric method}

The number of chelates per antibody, was determined in purified immunoconjugate by the mass spectrometry method (MALDI / TOF) (Siegel et al., 1991), using spectrophotometer Bruker Autoflex Speed (CEFAP-ICB/ USP). The molecular weight of the immunoconjugate, in different molar ratios, was obtained and the molecular weight of the antibody, equivalent to $150,000 \mathrm{~g} / \mathrm{mol}$, was subtracted. The difference corresponds to the chelating mass incorporated into Cetuximab. The chelating mass was divided by the molecular weight of the chelator (DTPA: $649.9 \mathrm{~g} / \mathrm{mol}$ ) and the result was expressed in a number of chelators coupled to the monoclonal antibody molecule.

\section{Stability of the Immunoconjugate}

Aliquots of $20 \mu \mathrm{L}$ of Cetuximab-DTPA $(5 \mathrm{mg} / \mathrm{mL})$, conjugated at molar ratios 10:1 and 20:1, were fractionated in $1.5 \mathrm{~mL}$ microcentrifuge tubes (Eppendorf, Brazil) and stored at $2-8^{\circ} \mathrm{C}$ and $-20^{\circ} \mathrm{C}$. An aliquot was taken weekly for analysis by HPLC, using the same system as described above.

\section{Radiolabeling development and analysis of specific activity}

Cetuximab-DTPA $(5 \mathrm{mg} / \mathrm{mL})$ was radiolabeled with different 111 -Indium activities $(0.185 \mathrm{MBq} / \mu \mathrm{L}-3.7 \mathrm{MBq} /$ 
$\mu \mathrm{L})$ (Nordion/Canada) in $0.25 \mathrm{M}$ ammonium acetate buffer $\mathrm{pH}$ 6.5, the reaction time was 30 minutes at $42{ }^{\circ} \mathrm{C}$ (Lewis, Raubitschek, Shively, 1994).

The radioimmunoconjugates were stored under refrigeration $\left(2-8{ }^{\circ} \mathrm{C}\right)$ for 48 hours and radiochemical purity was determined by CCD using $50 \mathrm{mM}$ EDTA and $0.1 \mathrm{M}$ ammonium acetate buffer $\mathrm{pH} 5.5$ - 6.0 as a mobile phase and then a glass fiber plate was impregnated with silica gel (ITLC-SG, Merck, Germany) as stationary phase. The distribution of radioactivity in the chromatographic plate was performed by radiochromatography (radio-TLC Imaging Scanner AR-2000, Eckert \& Ziegler / USA) (Chang, Smith, Lapi, 2013). The radiochemical purity was also evaluated by HPLC (Agilent, detector UV 190-300 nm) (Cooper, Sabbah, Mather, 2006; Chang, Smith, Lapi, 2013), using the same methodology as described above.

\section{Analysis of the stability of radiolabeled Cetuximab in human serum}

The radioimmunoconjugate $(18.5 \mathrm{MBq})$ was added to $1 \mathrm{~mL}$ of human serum. To obtain the human serum, 10 $\mathrm{mL}$ of blood was collected, without coagulant, from a healthy donor. The sample was then centrifuged at 1400 rpm for 10 minutes. After centrifugation, the fibrin clot was removed and a new centrifugation was performed to separate the serum. The samples were incubated at 37 ${ }^{\circ} \mathrm{C}, 350 \mathrm{rpm}$ for 72 hours. After 1, 24, 48 and 72 hours of incubation (Lewis, Raubitschek, Shively, 1994), the aliquots were analyzed by CCD as previously described.

\section{Binding of the radiolabeled Cetuximab to serum protein in vitro}

To determine the ${ }^{111}$ In-DTPA-Cetuximab binding to serum proteins, $18.5 \mathrm{MBq}$ of radiolabeled $\mathrm{mAb}$ was added to $1 \mathrm{~mL}$ of human serum in triplicate. After each incubation period, 1, 24, 48 and 72 hours, an aliquot (200 $\mu \mathrm{L})$ was withdrawn and ethanol was added $(1: 1 \mathrm{v} / \mathrm{v})$ to precipitate proteins. The sample was centrifuged at 10,000 rpm and the precipitate and supernatant radioactivity was determined using a gamma counter (D5002 Cobra II, Canberra Packard, USA) (Lewis, Raubitschek, Shively, 1994). The percent of serum protein binding was calculated (radioactivity of precipitate/radioactivity of precipitate + radioactivity of supernatant).

\section{RESULTS AND DISCUSSION}

Several immunotherapeutic agents are commercialized to receptor-specific cancer therapy, including Cetuximab. However, there are no radiopharmaceuticals available for RID or RIT in Brazil. In this work, methodologies of the conjugation of MAb to bifunctional chelators were studied and the effectiveness of radiolabeling methods of the immunoconjugates with indium-111 was evaluated in order to obtain radiopharmaceuticals for diagnostic application.

\section{Analysis of the antibody structural integrity}

The cetuximab integrity was confirmed by electrophoretic analysis on a polyacrylamide gel (Figure 2). Cetuximab analyzed samples (A1 to A3) were compared to the protein molecular standard, showing a molecular weight compatible with the antibody molecule.

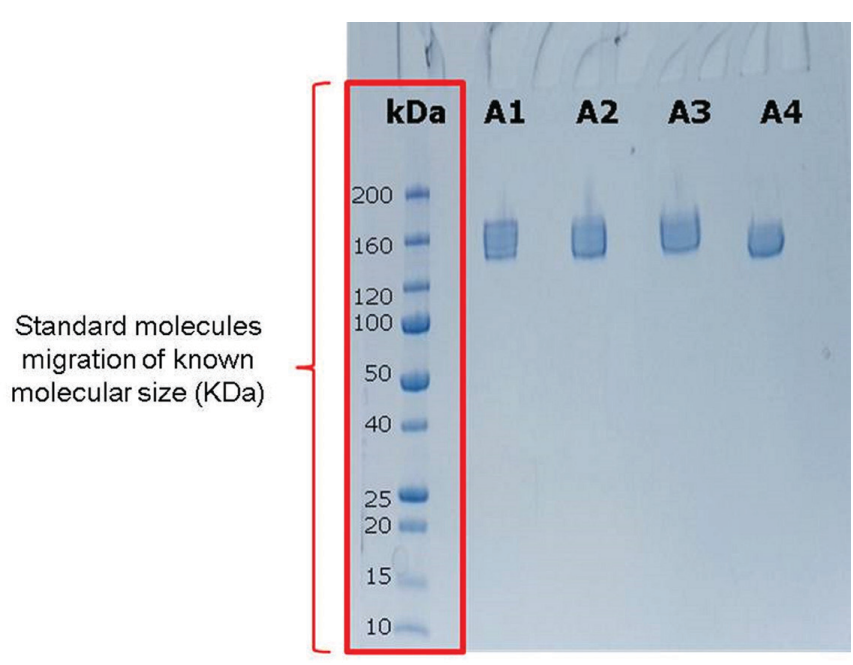

FIGURE 2 - Electrophoresis in 4-20\% Tris-Glicin gel: A1 / A3 (Cetuximab samples); A2 / A4 (Immunoconjugates).

\section{Cetuximab conjugation with p-SCN-Bn-DTPA}

Mabs labeled with radiometals are generally possible to prepare by the use of bifunctional chelating agents possessing a reactive portion for covalent attachment to proteins and a portion capable to bind tightly to the radiometal, thus forming a physiologically stable complex with the radionuclide. Such stability is essential for the agents used in RID and RIT, in order to reduce radiation damage and toxicity to normal organs and tissues (Rasaneh et al., 2010).

According to the chromatographic profile (Figure 3 ), the fractions corresponding to $4.0 \mathrm{~mL}, 4.5 \mathrm{~mL}, 5.0$ $\mathrm{mL}, 5.5 \mathrm{~mL}$ and $6.0 \mathrm{~mL}$, obtained from immunoconjugate purification process in PD-10, were selected since they do not have or present a minimal amount of the free 
chelator. Subsequently, the fractions were assembled in an ultracentrifugation tube, in order to eliminate the residual chelator and concentrate the immunoconjugate sample for radiolabeling procedure.
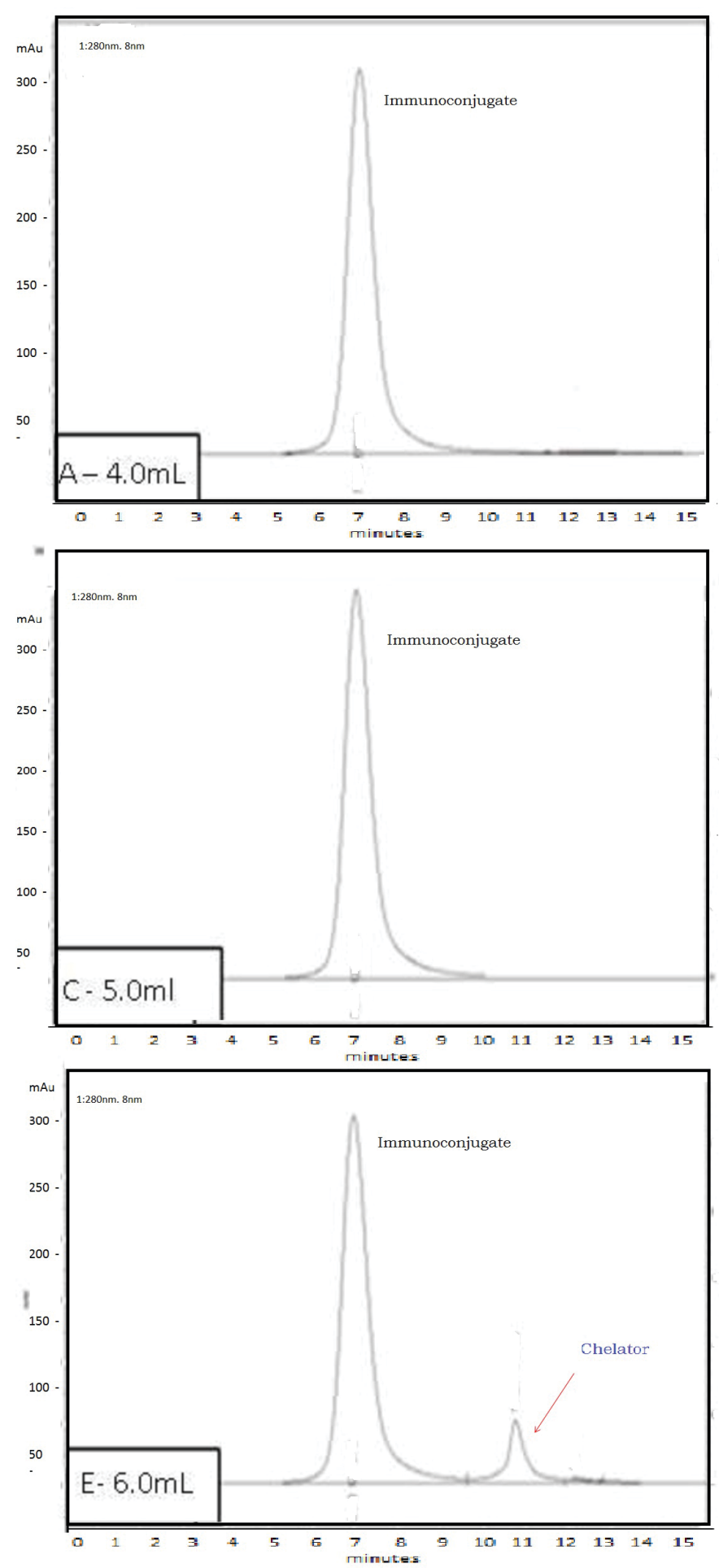

Cooper and colleagues (2006) used only concentrator tubes (ultrafiltration) for purification and concentration. This process has a disadvantage due to the high number of spin cycles, around eight cycles,
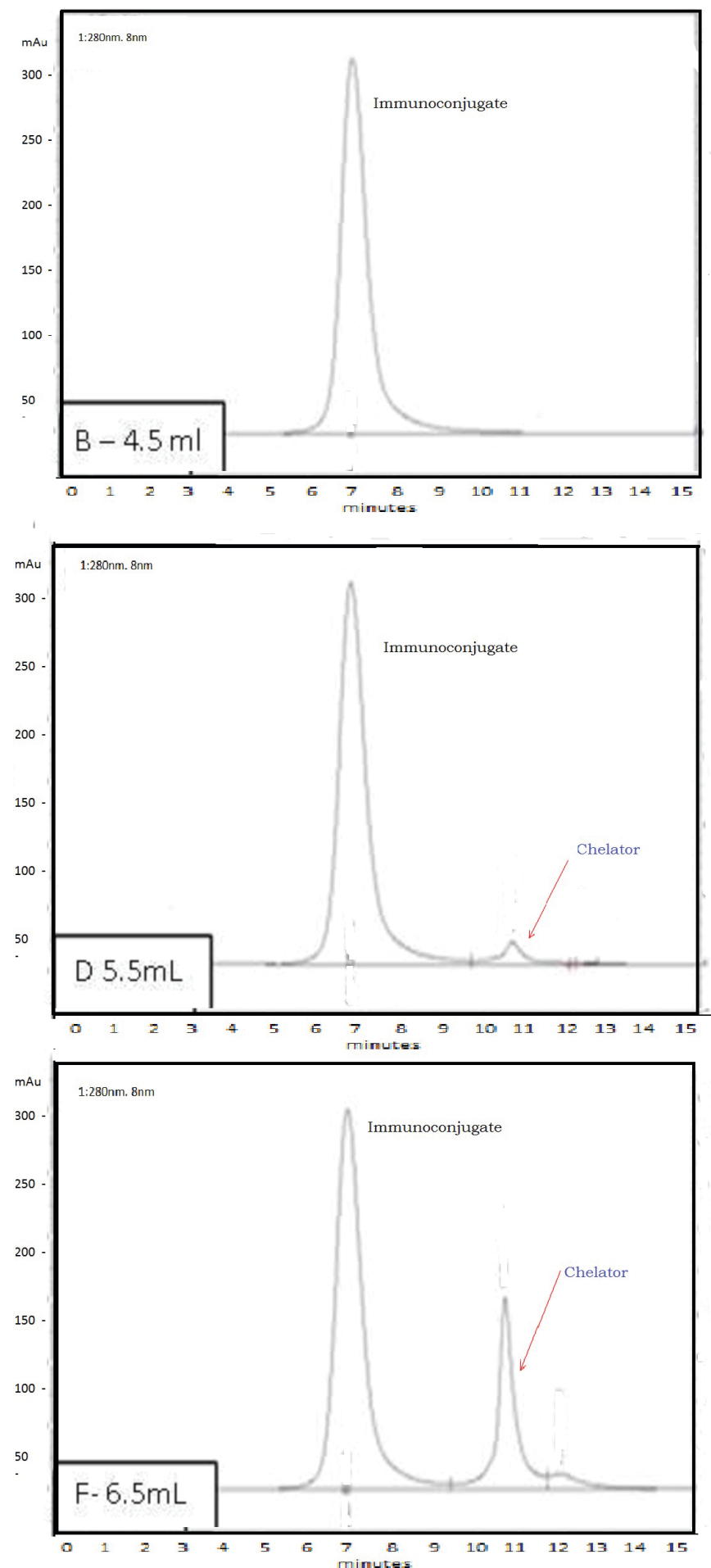

FIGURE 3 - HPLC Immunoconjugate chromatographic profile (UV, $280 \mathrm{~nm}$ ) after the purification process through size exclusion column (PD10), using 0.25 M ammonium acetate buffer pH 6.5 as eluent. A $(4.0 \mathrm{~mL})-\mathrm{C}(5.0 \mathrm{~mL})$ : the peak corresponds to the conjugated antibody; $\mathrm{D}(5.5 \mathrm{~mL})-\mathrm{F}(6.5 \mathrm{~mL})$ : the peaks correspond to the conjugated antibody and free chelator, retention time (RT) approximately 11 minutes. 
30 minutes each, to complete the purification of the immunoconjugate.

The procedure used in this work, with preliminary purification in a PD-10 column and further purification and concentration by ultrafiltration, reduced the time expended on purification by $50 \%$, since it took only two centrifugation cycles to concentrate the immunoconjugate. In addition, this method provided an immunoconjugate with purity exceeding $99 \%(\mathrm{~N}=15)$, as showed in HPLC profile (Figure 3 and $7 \mathrm{~A}$ ).

The retention time (RT) for the unconjugated antibody was compared with the RT of the immunoconjugate showing significant differences for both molar ratios: DTPA 10:1 ( $<<0.0001)$, DTPA 20:1 $(\mathrm{P}<0.0001)$ (Table I).

The same RT in HPLC profile was observed for the $\mathrm{mAb}$ conjugated with DTPA at both molar ratios 10:1 and 20:1, apparently indicating no greater incorporation of DTPA chelators to $\mathrm{mAb}$ when the molar ratio was doubled.

The immunoconjugate was analyzed for stability and the samples remained stable when stored at $-20{ }^{\circ} \mathrm{C}$, showing an single peak higher to $98 \%(\mathrm{~m}=98.7 \% \pm 0.9$; $\mathrm{N}=36$ ) in HPLC profile, that corresponds to the Cetuximab-DTPA (Figure 4).

TABLE I - HPLC analysis of the unconjugated antibody and immunoconjugate. Retention time (RT) in minutes. Results expressed as mean \pm SD. Chromatograms obtained by Shimadzu system

\begin{tabular}{ccc}
\hline Molar ratio & $\begin{array}{c}\text { RT cetuximab } \\
\text { (min) }\end{array}$ & $\begin{array}{c}\text { RT } \\
\text { immunoconjugate } \\
\text { (min) }\end{array}$ \\
\hline DTPA 10:1 & $7,33 \pm 0,26$ & $6,76 \pm 0,05$ \\
\hline DTPA 20:1 & $7,31 \pm 0,29$ & $6,72 \pm 0,03$ \\
\hline
\end{tabular}

\section{Analysis of the protein concentration of the immunoconjugate}

The determination of the protein concentration after conjugation and purification is essential to calculate the radiopharmaceutical specific activity and analysis of radiolabeling conditions.

The results of protein concentrations for the Cetuximab-DTPAimmunoconjugate, produced with molar ratios $10: 1$ and $20: 1$ were $4.43 \pm 0.24 \mathrm{mg} / \mathrm{mL}$ and $4.54 \pm$ $0.22 \mathrm{mg} / \mathrm{mL}$, respectively. Considering an initial antibody concentration of $5 \mathrm{mg} / \mathrm{mL}$, it obtained a protein recovery above $83 \pm 1.42 \%(\mathrm{~N}=15)$. These results demonstrated that the method applied for purification of immunoconjugate was more reproducible than the method described in the
Immunoconjugate Stability - mAb-DTPA - [1:10]

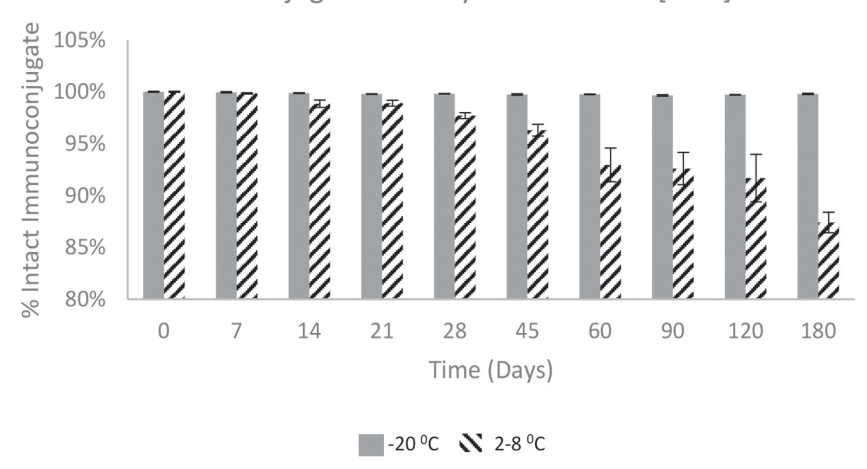

Immunoconjugate Stability - mAb-DTPA - [1:20]

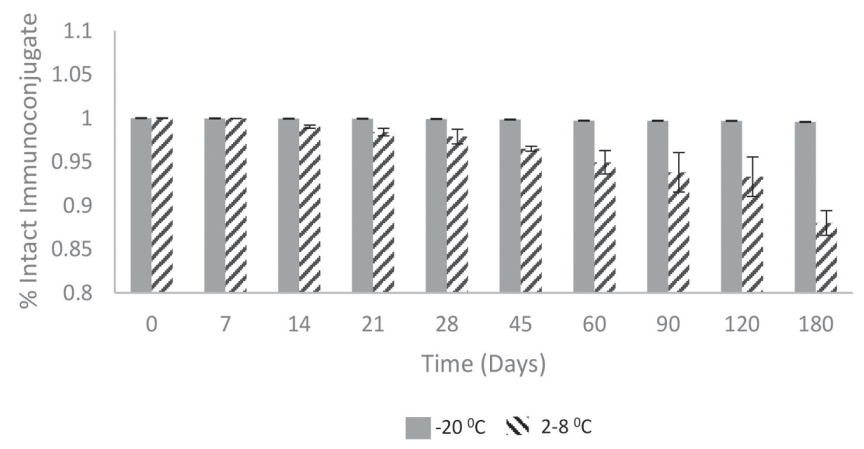

FIGURE 4 - HPLC stability study of immunoconjugate at different DTPA:mAb molar ratios and time after conjugation (days).

literature (Cooper, Sabbah, Mather, 2006), which results are variable due to the loss of protein $(10-50 \%)$.

\section{Determination of number of chelators per antibody}

The optimal number of chelators per antibody must be achieved in the process of conjugation, in order to guarantee the stability of the immunoconjugate, under storage and after radiolabeling, to preserve the antibody immunoreactivity. The immunoconjugate molecular weight was determined by mass spectrometry (MALDI/TOF) (Figure 5), and the number of chelators coupled to the antibody were calculated through the difference of unconjugated antibody molecular weight (Table II). The mass spectrometry is the most accurate method for determining chelators coupled to mAbs. The results demonstrated that even with a higher amount of chelator (20:1), there was no change in the number of p-SCN-Bn-DTPA chelators coupled to Cetuximab ( $p$-value $>0.06$ ). Approximately 6-7 chelators/ $\mathrm{mAb}$ were incorporated, confirming the results obtained in HPLC, in which there was no significant difference between the retention times of the peaks generated with the immunoconjugate molar ratio $10: 1$ or $20: 1$. 


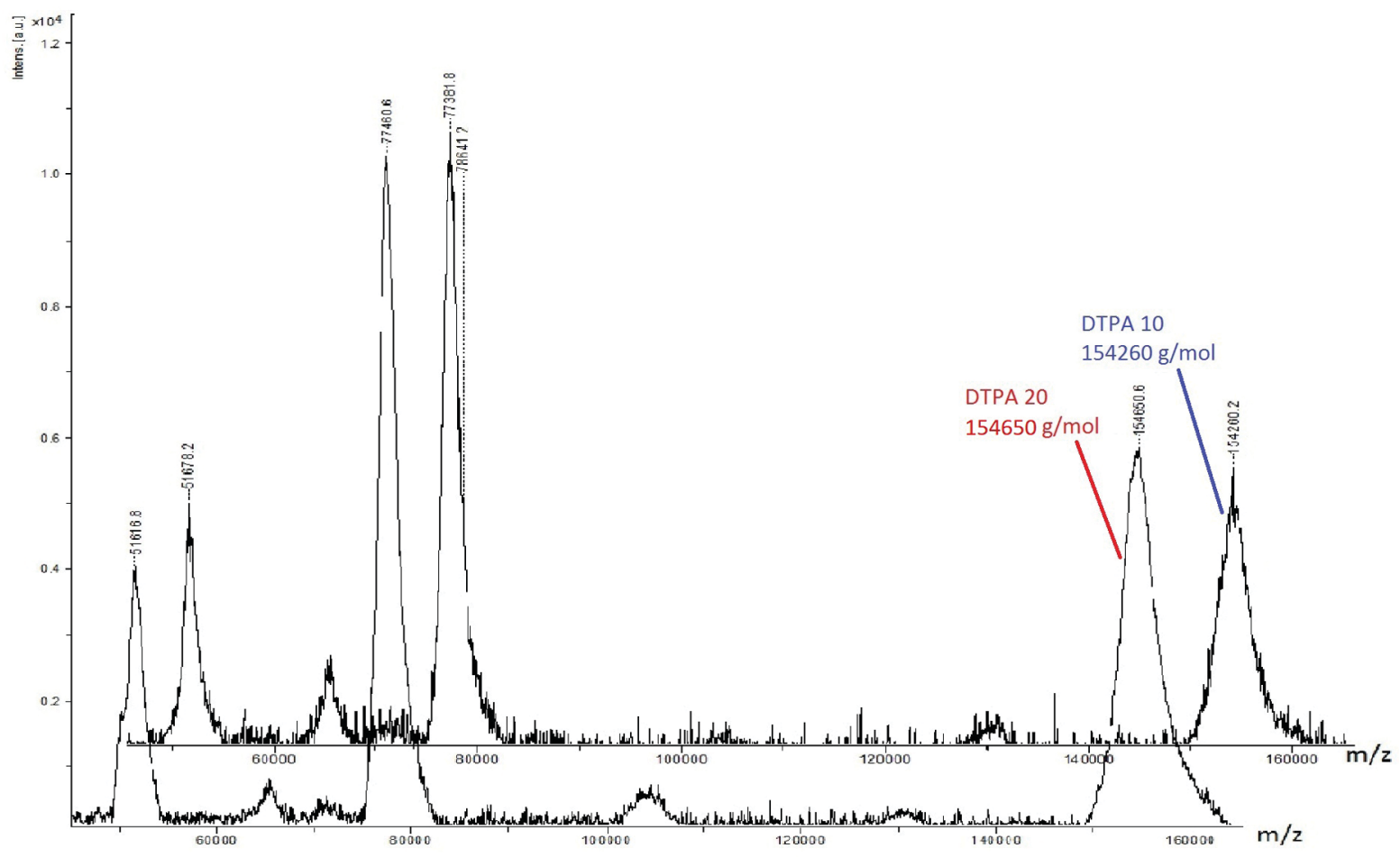

FIGURE 5 - MALDI/TOF immunoconjugate spectrum (spectrometer Bruker Autoflex Speed, CEFAP ICB / USP). Molecular weight (MW) of Cetuximab-DTPA produced at different molar ratios: DTPA $10 \mathrm{MW}$ was $154.260 \mathrm{~g} / \mathrm{mol}$; DTPA $20 \mathrm{MW} 154.650 \mathrm{~g} / \mathrm{mol}$.

TABLE II - Determination of number of chelators per antibody by spectrometry Autoflex MALDI-TOF/TOF

\begin{tabular}{ccccc}
\hline $\begin{array}{c}\text { Molecular Weight } \\
\text { Chelator DTPA (g/mol) }\end{array}$ & $\begin{array}{c}\text { Chelation } \\
\text { Molar Ratio }\end{array}$ & $\begin{array}{c}\text { MALDI/TOF } \\
\text { Molecular Weight } \\
\text { (g/mol) }\end{array}$ & $\begin{array}{c}\text { Difference } \\
\text { [imunoconjugated } \\
(\mathbf{g} / \mathbf{m o l})-C e t u x i m a b \\
(\mathbf{1 5 0 . 0 0 0 g} / \mathbf{m o l})]\end{array}$ & $\begin{array}{c}\text { Number } \\
\text { Chelator/mAb }\end{array}$ \\
\hline 649.9 & DTPA 10 & 154260 & 4260 & 6.6 \\
649.9 & DTPA 20 & 154650 & 4650 & 7.2 \\
\hline
\end{tabular}

\section{Radioimmunoconjugate development and analysis of specific activity}

There are difficulties in radiolabeling conjugated mAbs, since the presence of trace metals (contaminants) can compete with the radiometal, interfering directly with the radiolabeling and consequently reducing radiochemical yield. This fact requires pre purification of all reagents employed in the labeling step for removing metals. Moreover, the purification and concentration of the conjugated protein are important steps to remove the excess chelating agent added for the conjugation process.

Figure 6 shows the percentage of radiochemical purity (\% PR) for radioimmunoconjugate at different specific activities, obtained by TLC analysis immediately,
24 and 48 hours after labeling procedure. The samples were stored at $2-8{ }^{\circ} \mathrm{C}$. The specific activity of 185 $\mathrm{MBq} / \mathrm{mg}(5 \mathrm{mCi} / \mathrm{mg})$ was adopted as reference since it showed superior activity, with higher radiochemical purity yields (Figure 7). The average of radiochemical purity, immediately after labeling, was greater than $95 \%$ for the specific activity $74 \mathrm{MBq} / \mathrm{mg}(95.9 \% \pm$ $1.8), 111 \mathrm{MBq} / \mathrm{mg}(96.2 \% \pm 1.6)$ and $185 \mathrm{MBq} / \mathrm{mg}$ $(97.3 \% \pm 1.3)$. A significant difference in decreasing radiochemical purity, was observed $(\mathrm{P}=0.036)$, when comparing the results of radiochemical purity over per time. Nevertheless, radioimmunoconjugates with specific activity of $111 \mathrm{MBq} / \mathrm{mg}$ and $185 \mathrm{MBq} / \mathrm{mg}$ were stable for 48 hours, which allows a possible distribution of the radiopharmaceutical, even in more distant regions of the production center. 


\section{\% Radiochemical Purity - mAb-DTPA - [111]In (1:20)}

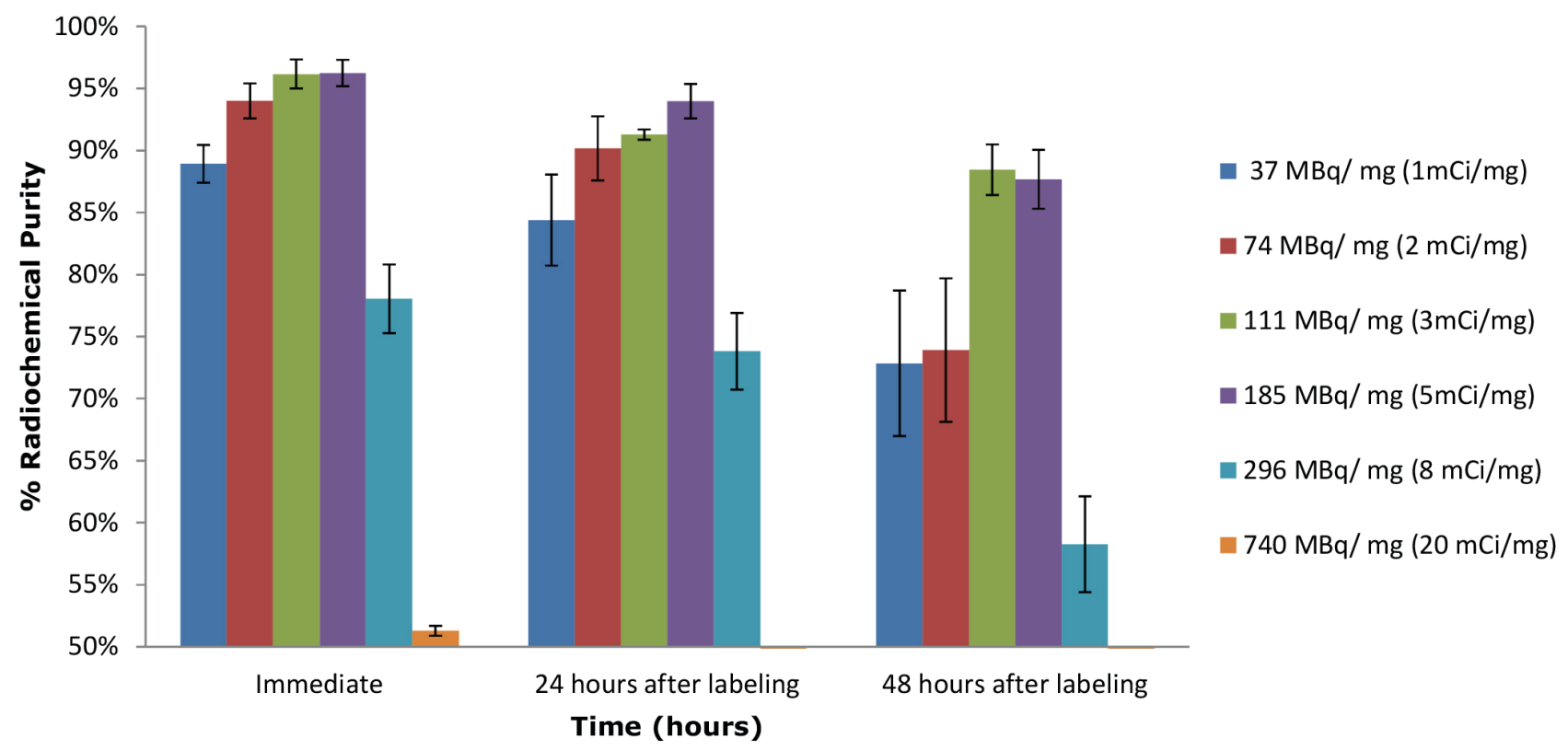

FIGURE 6 - Radioimmunoconjugate stability study (48 hours) - Radiochemical purity analysis of Cetuximab-DTPA (20:1) radiolabeled with indium-111, at different specific activities $(\mathrm{MBq} / \mathrm{mg})$. Results obtained by $\mathrm{CCD}(\mathrm{n} \geq 3)$.
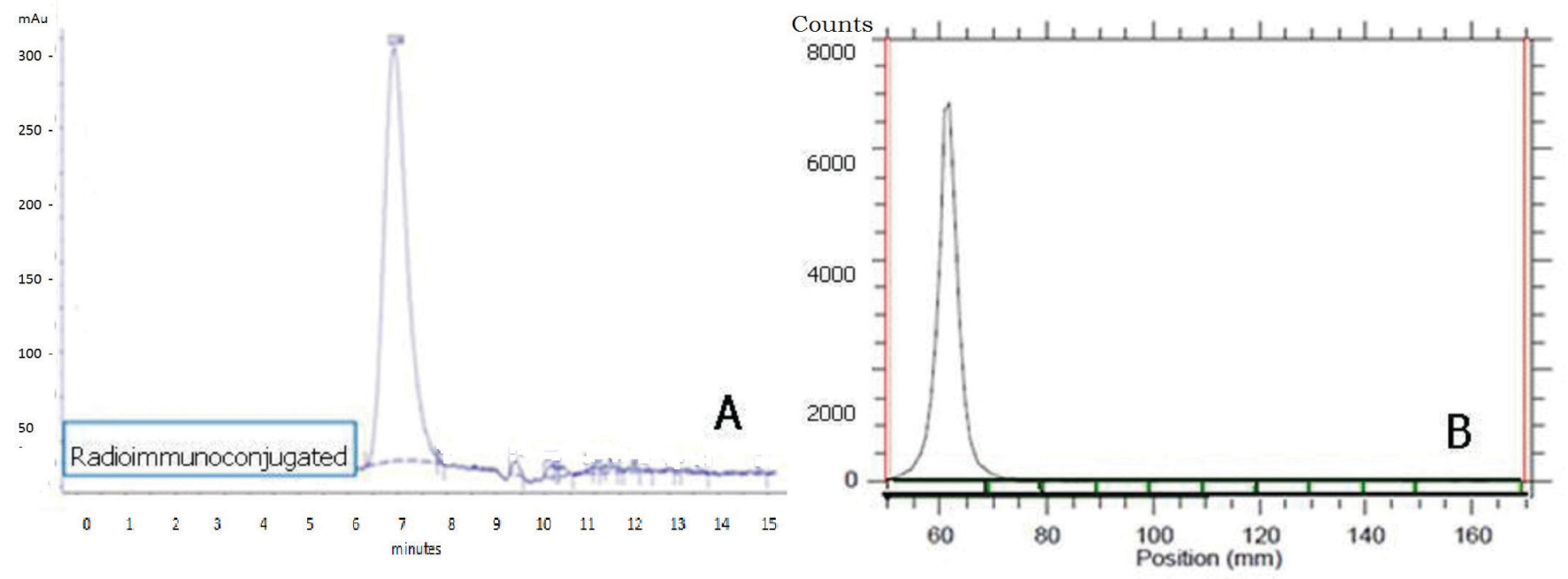

FIGURE 7 - HPLC chromatographic profile of the radioimmunoconjugate, $185 \mathrm{MBq} / \mathrm{mg}$ (A). TLC scanner chromatographic profile of the radioimmunoconjugate, $185 \mathrm{MBq} / \mathrm{mg}(\mathrm{B})$.

\section{Stability analysis of radiolabeled Cetuximab in human serum and binding to serum protein in vitro}

Considering the results obtained, the specific activity of $185 \mathrm{MBq} / \mathrm{mg}(5 \mathrm{mCi} / \mathrm{mg})$ has been used to evaluate the stability of the radiolabeled Cetuximab in human serum. According to stability study in human serum, no bound plasma proteins ${ }^{111}$ In-DTPA-Cetuximab degraded in 48 hours, possibly due to plasma enzyme activity (Figure 8A).
In addition, a high fraction of radiolabeled $\mathrm{mAbs}$ bound to serum protein (Figure $8 \mathrm{~B}$ ), which should contribute to the slow blood clearance in vivo.

Diagnostic information can assist in the selection of patients, and can potentially predict the risk-benefit balance of a planned therapy with radionuclides through the dosimetric analysis. In addition, the imaging data can evaluate the therapeutic response to a particular treatment, aiming specifically to guide the planning and therapeutic conduction (Denardo, Sysko, Denardo, 2006). 

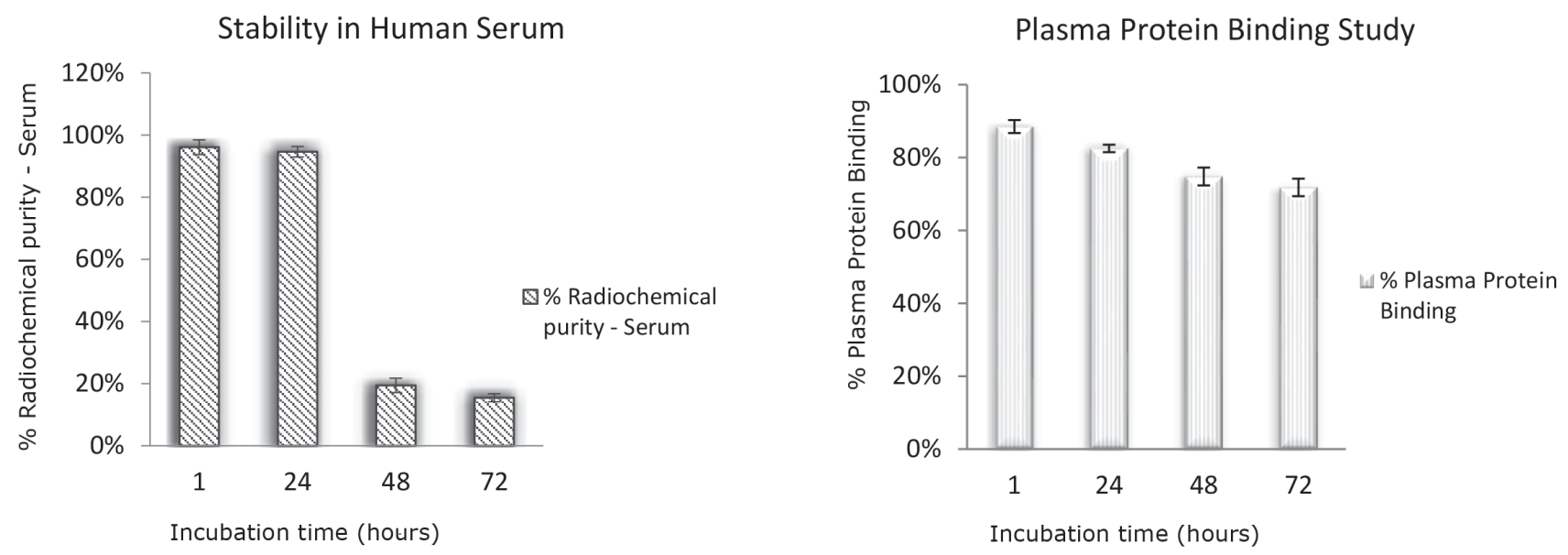

FIGURE 8 - Radioimmunoconjugate stability in human serum (A); \% protein binding (B). Results expressed as mean \pm SEM ( $>3$ ).

This study demonstrated that ${ }^{111}$ In-DTPA-Cetuximab has been efficiently developed. The conjugation with the chelator DTPA was successful and the immunoconjugate presented stability suitable for the routine production of radiopharmaceuticals. The labeled antibody was produced with high radiochemical yield and high specific activity, showing that ${ }^{111}$ In-DTPA-Cetuximab is feasible to produce for its application in immuno-SPECT for the diagnosis of head-and- neck cancers and colorectal carcinoma. therefore, encouraged the progress of the clinical studies.

\section{ACKNOWLEDGMENTS}

The author is grateful to scientific cooperation of Marycel Rosa Felisa Figols Barboza and the National Commission of Nuclear Energy - CNEN, for financial support.

\section{REFERENCES}

Brady ED, Chong HS, Milenic DE, Brechbiel MW. Development of a spectroscopic assay is difunctional ligand-protein conjugates based on copper. Nucl Med Biol. 2004;31(6):795-802.

Chang AJ, Smith RA, Lapi S. Development and characterization of $89 \mathrm{Zr}$-Labeled panitumumab is immuno-positron emission tomographic imaging of the epidermal growth factor receptor. Mol Imaging. 2013;12(1):17-27.

Cooper MS, Sabbah E, Mather SJ. Conjugation of chelating agents to proteins and radiolabeling with trivalent metallic isotopes. Nature Protocols. 2006;1(1):314-317.

Denardo GL, Sysko VV, Denardo SJ. Cure of incurable lymphoma. Int J Radiat Oncol Biol. 2006;66(Suppl.2):46-56.
Divgi CR, Welt S, Kris M, Real FX, Yeh SD, Gralla R, et al. Phase I and imaging trial of indium-111 labeled antiepidermal growth factor receptor monoclonal antibody 225 in Patients with squamous cell lung carcinoma. J Natl Cancer Inst.1991;83(2):97-104.

Doerr RJ1, Abdel-Nabi H, Baker JM, Steinberg S. Detection of primary colorectal cancer with indium 111 monoclonal antibody B72.3. Arch Surg. 1990;125(12):1601-5.

Evans HL, Nguyen Q, Carroll LS, Kaliszczak M, Twyman FJ, Spivey AC, et al. A bioorthogonal 68 Ga-labeling strategy is alive rapidin imaging. Chem Commun. 2014;50:9557-60.

Hoeben BAW, Molkenboer-Kuenen JDM, Oyen WJG, Peeters WJM, Bussink J, Boerman OC. Radiolabeled cetuximab: dose optimization for epidermal growth factor recept or imaging in the head-and-neck squamous cell carcinoma model. Int J Cancer. 2011;129(4):870-878.

Instituto Nacional do Cancer. INCA. Cancer incidence in Brazil - estimate 2014. [cited 2016 May 11]. Available from: http:// www.inca.gov.br/estimativa/2014/estimativa-24042014.pdf.

Jalilian AR, Beiki D, Hassanzadeh-Rad A, Eftekhari A, Geramifar P, Eftekhari M. Production and clinical applications of radiopharmaceuticals and medical radioisotopes in Iran. Sem Nucl Med. 2016;46(4):340-358.

Laemmli, UK. Cleavage of structural proteins during the assembly of the head of bacteriophage T4. Nature. 1970;227:680-685. 
Lewis MR, Raubitschek A, Shively JE. The facile, water-soluble method for modification of proteins with DOTA. Use of elevated temperature and optimized $\mathrm{pH}$ to Achieve high specific activity and high chelate stability in radiolaleled immunoconjugates. Bioconjug Chem. 1994;5(6):565-576.

Mccarron PA, Olwll SA, Marouf WM, Buick RJ, Walker B, Scott CJ. Antibody conjugates and therapeutic strategies. Mol Interv. 2005;5(6):368-380.

Morris MJ, Divgi CR, Pandit-Taskar N, Batraki M, Warren N, Nacca A, Smith-Jones P, et al. Pilot trial of unlabeled and indium-111-labeled anti-prostate-specific membrane antigen antibody J591 for castrate metastatic prostate cancer. Clin. Cancer Res. 2005;11(20):7454-61.

Niu G, Sun X, Cao Q, Courter D, Koong A, Le QT, et al. Cetuximab-based immunotherapy and radioimmunotherapy of head and neck squamous cell carcinoma. Clin Cancer Res. 2010;16(7):2095-2105.

Perik PJ, Lub-de HMN, Gietema JA, van der Graaf WT, Korte MA, Jonkman S, et al. Indium-111-labeled trastuzumab scintigraphy in Patients with human epidermal growth factor receptor-2 positive metastatic breast cancer. J Clin Oncol. 2006;24(15):2276-2282.

Ping LW, Meyer LA, Capretto DA, Sherman CD, Anderson CJ. Receptor-binding, Biodistribution, and metabolism studies of $64 \mathrm{Cu}$-DOTA-cetuximab, PET-imaging agent for epidermal receptor-positive tumors growth-factor. Cancer Biother Radiopharm. 2008;23(2):158-171.

Rasaneh S, RajabiI H, Babaei MH, Daha FJ. 177Lu labeling of Herceptin and preclinical validation as a new radiopharmaceutical for radioimmunotherapy of breast cancer. Nucl Med Biol. 2010;37(8):949-955.

Reilly RM, Lam K, Chan C, Levine M. Advancing Molecular novel imaging agents from preclinical studies to first-in-humans Phase I clinical trials in academia-a roadmap for overcoming perceived barriers. Bioconjug Chem. 2015;26(4):625-32.
Schechter NR, Wendt RE, Yang DJ, Azhdarinia A, Erwin WD, Stachowiak AM, et al. Radiation dosimetry of 99mTc-labeled C225 in Patients with squamous cell carcinoma of the head and neck. J Nucl Med. 2004;45(10):1683-1687.

Scott AM, Tebbutt N, Lee FT, Cavicchiolo T, Liu Z, Gill S, et al. The phase I and Biodistribution pharmacokinetic trial of humanized monoclonal antibody Hu3s193 in Patients with advanced epithelial cancers que express the Lewis Y antigen. Clin Cancer Res. 2007;13(11):3286-92.

Siegel MM, Hollander IJ, Hamann PR, James JP, Hinman L, Smith BJ. Matrix-assisted laser desorption-UV/ionization mass spectrometric analysis of monoclonal antibodies for the determination of carbohydrate, conjugated chelator and conjugated drug content. Anal Chem. 1991;63(21):2470-81.

Sihver W, Pietzsch J, Krause M, Baumann M, Steinbach J, Pietzsch H. Radiolabeled targeted EGFR cetuximab conjugates for cancer diagnostics and therapy. Pharmaceuticals. 2014;7(3):311-38.

Strome SE, Sausville EA, Mann D. Mechanistic perspective of monoclonal antibodies in cancer therapy beyond target-related effects. Oncologist. 2007;12(9):1084-95.

Van de Watering FJC, Rijpkema M, Perk L, Brinkmann U, Oyen WJG, Boerman OC. Zirconium-89 labeled antibodies: a new tool for molecular imaging in cancer patients. BioMed Res Int. 2014;14:203601.

Wiechec E, Hansson KT, Alexandersson L, Jönsson JI, Roberg K. Hypoxia Mediates Differential Response to Anti-EGFR Therapy in HNSCC Cells. Int J Mol Sci. 2017;943(18):1-12.

Received for publication on $07^{\text {th }}$ February 2017 Accepted for publication on $15^{\text {th }}$ May 2017 